\title{
Semi-heavy tails
}

\author{
Edward Omey ${ }^{\mathrm{a}}$, Stefan Van Gulck ${ }^{\mathrm{a}}$, and Rein Vesilo ${ }^{\mathrm{b}}$ \\ ${ }^{a}$ Faculty of Economics and Business, KU Leuven, Campus Brussels, Warmoesberg 26, 1000 Brussels, Belgium \\ ${ }^{\mathrm{b}}$ Department of Engineering, Macquarie University, NSW 2109, Australia \\ (e-mail: edward.omey@kuleuven.be; stefan.vangulck@kuleuven.be; rein.vesilo@mq.edu.au)
}

Received January 25, 2017; revised August 15, 2018

\begin{abstract}
In this paper, we study properties of functions and sequences with a semi-heavy tail, that is, functions and sequences of the form $w(x)=\mathrm{e}^{-\beta x} f(x), \beta>0$, resp., $w_{n}=c^{n} f_{n}, 0<c<1$, where the function $f(x)$, resp., the sequence $\left(f_{n}\right)$, is regularly varying. Among others, we give a representation theorem and study convolution properties. The paper includes several examples and applications in probability theory.
\end{abstract}

MSC: 26A12, 33B99, 60K05

Keywords: semi-heavy tail, regular variation, convolutions, asymptotic behaviour, subordination

\section{Introduction}

Throughout the paper we use either discrete random variables (r.vs) $X$ or continuous r.vs $X$ that have a density. In the discrete case, we assume that $X$ has a probability mass function (p.m.f.) $w_{n}=\mathbf{P}(X=n), n \geqslant 0$. In the continuous case, we assume that $X$ has a distribution function (d.f.) $F(x)$ and a probability density function (p.d.f.) $f(x)$. In any case the distribution function (d.f.) is given by $F(x)=\mathbf{P}(X \leqslant x)$, and the tail distribution is given by $\bar{F}(x)=1-F(x)$.

The d.f. is called heavy tailed if for all $\lambda>0, \bar{F}(x)$ satisfies $\lim _{x \rightarrow \infty} \mathrm{e}^{\lambda x} \bar{F}(x)=\infty$. The d.f. has a longtailed distribution if $\bar{F}$ satisfies

$$
\lim _{x \rightarrow \infty} \frac{\bar{F}(x+y)}{\bar{F}(x)}=1 \quad \forall y \in \Re .
$$

The d.f. has a fat tail if $\bar{F}$ is of the form $\bar{F}(x)=h(x)$, where $h(x)$ is regularly varying with index $\alpha<0$. The r.v. $X$ has a fat density if $f(x)=h(x)$, where $h$ is regularly varying. Recall that a positive measurable function $h$ is regularly varying with (real) index $\alpha$ if

$$
\lim _{x \rightarrow \infty} \frac{h(x y)}{h(x)}=y^{\alpha}, \quad y>0 .
$$

Notation: $h \in \operatorname{RV}(\alpha)$. For the basic properties of the class $\operatorname{RV}(\alpha)$, we refer to Bingham et al. [4] or Geluk and de Haan [10]. It can be proved that if $h \in \operatorname{RV}(\alpha)$, then (1.1) holds locally uniformly in $y>0$. Then it follows 
that $h \in \mathrm{L}$, where $\mathrm{L}$ is the class of positive measurable functions $h$ for which

$$
\lim _{x \rightarrow \infty} \frac{h(x+y)}{h(x)}=1 \quad \forall y \in \Re .
$$

Note that $h(x) \in \mathrm{L}$ iff $h(\log x) \in \mathrm{RV}(0)$. It can be proved that if $h \in \operatorname{RV}(\alpha)$, then

$$
\forall \beta>0, \quad \lim _{x \rightarrow \infty} x^{\beta-\alpha} h(x)=\infty \quad \text { and } \quad \lim _{x \rightarrow \infty} x^{-\beta-\alpha} h(x)=0 .
$$

We say that a sequence $\left(z_{n}\right)$ is regularly varying with index $\alpha$ if $z_{n}>0$ for $n$ large and if it satisfies $z_{[x y]} / z_{[x]} \rightarrow y^{\alpha}, y>0$. Notation: $\left(z_{n}\right) \in \operatorname{RS}(\alpha)$. If $\left(z_{n}\right) \in \operatorname{RS}(\alpha)$, then it follows that $\left(z_{n}\right)$ is in the class LS where LS is the class of sequences with $z_{n}>0$ for $n$ large and $z_{n+1} / z_{n} \rightarrow 1$. Notation: $\left(z_{n}\right) \in \operatorname{LS}$.

If $X$ has a fat tail, then $\bar{F} \in \operatorname{RV}(-\alpha)$ with $\alpha>0$, and then we have

$$
\forall \delta>0, \quad \lim _{x \rightarrow \infty} x^{\alpha-\delta} \bar{F}(x)=0 \quad \text { and } \quad \lim _{x \rightarrow \infty} x^{\alpha+\delta} \bar{F}(x)=\infty .
$$

If the distribution of $X$ is long-tailed, then we have $\bar{F} \in \mathrm{L}$ or equivalently $\bar{F}(\log x) \in \mathrm{RV}(0)$. In this case, it follows that, for all $\delta>0, \lim _{x \rightarrow \infty} \mathrm{e}^{\delta x} \bar{F}(x)=\infty$. Throughout the paper, the asymptotic relations hold as $x \rightarrow \infty$ or $n \rightarrow \infty$, unless stated otherwise.

In this paper, we study, in general, functions and sequences that have a semi-heavy tail. In the rest of this section, we provide the key definitions used in the paper and give some examples of continuous and discrete probability distributions with the semi-heavy property. Section 2 begins with the important representation results used (Section 2.1) followed by asymptotic tail results for functions and sequences in Section 2.2. Section 2.3 gives the main convolution results developed in the paper. Applications of the results in the paper to subordinated random variables and queueing are given in Section 3, and concluding remarks are given in Section 4.

\subsection{Definition}

A sequence $\left(w_{n}\right)$ is called a semi-heavy tailed sequence if $w_{n}>0$ for $n$ large and if $w_{n}$ is of the form $w_{n}=c^{n} f_{n}, 0<c<1$, where $\left(f_{n}\right) \in \operatorname{RS}(\alpha)$ with index $\alpha \in \Re$. Notation: $\left(w_{n}\right) \in \operatorname{SHS}(c, \alpha)$.

A function $w(x)$ is called a semi-heavy tailed function if $w(x)>0$ for $x$ large and if $w(x)$ is of the form $w(x)=\mathrm{e}^{-\beta x} f(x), \beta>0$, where $f \in \operatorname{RV}(\alpha)$ with index $\alpha \in \Re$. Notation: $w \in \operatorname{SHF}(\beta, \alpha)$.

Using (1.3), it turns out that, for semi-heavy tailed sequences and functions, we have $w_{n} \rightarrow 0$, resp., $w(x) \rightarrow 0$, and it is why we use the word "tail" in the definition. Note that in the definition we do not assume that $\left(w_{n}\right)$ is a p.m.f. or that $w(x)$ is a p.d.f. or a distribution function. From the definition and (1.2) it is also clear that

$$
\lim _{x \rightarrow \infty} \frac{w(x+y)}{w(x)}=\mathrm{e}^{-\beta y} \quad \forall y \in \Re,
$$

and we have $w(\log x) \in \mathrm{RV}(-\beta)$. The class of positive measurable functions that satisfy (1.4) are denoted by $\mathrm{L}(\beta)$.

For sequences $\left(w_{n}\right) \in \operatorname{SHS}(c, \alpha)$, we have

$$
\lim _{n \rightarrow \infty} \frac{w_{n+1}}{w_{n}}=c .
$$

Sequences satisfying (1.5) are denoted by $\operatorname{LS}(c)$. Note that if $w \in \operatorname{SHF}(\beta, \alpha)$, then we also have that

$$
\lim _{x \rightarrow \infty} \mathrm{e}^{\delta x} w(x)= \begin{cases}0, & \delta<\beta, \\ \infty, & \delta>\beta .\end{cases}
$$


This means that the functions $w(x)$ are "thinner" than any power law and "heavier" than any normal law. Functions and sequence as defined before therefore are called semi-heavy functions and sequences.

It is useful to mention that semi-heavy tails appeared in modelling log increments of asset prices; see Barndorff-Nielsen [3], Schoutens [18], or Albin and Sunden [1]. Some examples follow in the next subsection.

\subsection{Examples}

There are many examples from probability theory.

\subsubsection{Continuous probability distributions}

1. The generalized inverse Gaussian distribution has been introduced by Halphen (cf. Seshadri [19]) and was reinvented and studied by Barndorff-Nielsen [3]; see also Borak et al. [5] and Prause [16]. Its density is of the following form:

$$
f_{G}(x)=C x^{\lambda-1} \exp \left(-\frac{1}{2}\left(\frac{a}{x}+b x\right)\right), \quad x>0,
$$

where $a>0$ and $b \geqslant 0$ if $\lambda<0, a>0$ and $b>0$ if $\lambda=0$, and $a \geqslant 0$ and $b>0$ if $\lambda>0$. Clearly, we have $f_{G}(x) \sim C x^{\lambda-1} \exp (-b x / 2)$ and $f_{G} \in \operatorname{SHF}(b / 2, \lambda-1)$.

2. A hyperbolic density has the form

$$
f_{H}(x)=C \exp \left(-\alpha \sqrt{\delta^{2}+(x-\mu)^{2}}+\beta(x-\mu)\right),
$$

where $x \in \Re, \alpha>\beta$; cf. Eberlein and Keller [7], Prause [16], and Borak et al. [5]. Clearly, we have $f_{H}(x) \sim C e^{\mu(\alpha-\beta)} \mathrm{e}^{-(\alpha-\beta) x}$ and $f_{H} \in \operatorname{SHF}(\alpha-\beta, 0)$.

3. The following density was introduced by Lindley [13]:

$$
f_{L}(x)=\frac{\theta^{2}}{1+\theta}(1+x) \mathrm{e}^{-\theta x}, \quad x>0, \theta>0 .
$$

Clearly, $f_{L}(x)=\mathrm{e}^{-\theta x} f(x)$, where $f(x)=\theta^{2}(1+\theta)^{-1}(1+x) \in \mathrm{RV}(1)$. Let $F_{L}$ denote the d.f. corresponding to $f_{L}$. Nadarajah et al. [14] study d.fs of the form $F_{N}(x)=F_{L}^{\alpha}(x), \alpha>0$. It can be observed that $\bar{F}_{N}(x) \sim \alpha \bar{F}_{L}(x)$. Zakerzadeh and Dolati [21] considered densities of the form

$$
f_{Z}(x)=C x^{\alpha-1}(a+b x) \mathrm{e}^{-\theta x}, \quad \alpha, a, b, \theta, x>0 .
$$

This density generalizes the Lindley and the gamma density. Clearly, $f_{Z} \in \operatorname{SHF}(\theta, \alpha)$.

\subsubsection{Discrete probability distributions}

1. We present an inverse Gaussian type of sequences as follows: $w_{n}=C n^{\alpha} \mathrm{e}^{-(a / n+b n)}, n \geqslant 1, a \geqslant 0, b>0$, $\alpha \in \Re$. Clearly, we have $\left(w_{n}\right) \in \operatorname{SHS}(c, \alpha)$ with $c=\mathrm{e}^{-b}$ and $f_{n} \sim C n^{\alpha} \in \operatorname{RS}(\alpha){ }^{1}$

2. A hyperbolic type of sequences is given by $w_{n}=C \exp \left(-\alpha \sqrt{a+n^{2}}+\beta n\right), n \geqslant 0, \alpha>\beta$. In this case, we obtain that $\left(w_{n}\right) \in \operatorname{SHS}(c, 0)$ with $c=\mathrm{e}^{-(\alpha-\beta)}$.

3. A discrete Lindley distribution can be defined as follows:

$$
p_{n}=C n^{\alpha-1}(a+b n) \mathrm{e}^{-\beta n}, \quad \alpha, a, b, \beta>0, n \geqslant 1 .
$$

Here we have $\left(p_{n}\right) \in \operatorname{SHS}(\beta, \alpha)$.

\footnotetext{
${ }^{1}$ For ease of use, but with a slight abuse of convention, by expressions of the form $a_{n} \sim b_{n} \in \operatorname{RS}(\alpha)$ we mean that $a_{n} \sim b_{n}$ and $\left(a_{n}\right) \in \operatorname{RS}(\alpha)$.
} 
4. Suppose that $X_{1}$ has a geometric distribution, that is, $\mathbf{P}\left(X_{1}=n\right)=p q^{n-1}, 0<p<1, q=1-p, n \geqslant 1$. Clearly, we have $\mathbf{P}\left(X_{1}=n\right)=q^{n} f_{n}$, where $f_{n}=p / q$. In general, suppose that $X_{k}(k \geqslant 1)$ has a negative binomial distribution:

$$
\mathbf{P}\left(X_{k}=n\right)=\left(\begin{array}{l}
n-1 \\
k-1
\end{array}\right) p^{k} q^{n-k}, \quad n \geqslant k .
$$

It follows that $\mathbf{P}\left(X_{k}=n\right)=q^{n} f_{n}$, where

$$
f_{n} \underset{n \rightarrow \infty}{\sim} \frac{n^{k-1}}{(k-1) !} \frac{p^{k}}{q^{k}} \in \operatorname{RS}(k-1)
$$

5. The generalized logarithmic series distribution is defined by the sequence

$$
p_{n}=\frac{\Gamma(\beta n)}{n ! \Gamma(\beta n-n+1)} \frac{\theta^{n}(1-\theta)^{\beta n-n}}{-\log (1-\theta)}
$$

with $\beta \geqslant 1$ and $0<\theta<\beta^{-1}$; see Hansen and Willekens [11]. For $\beta=1$, we find back the usual logarithmic p.d.f.

$$
p_{n}=\frac{-1}{\ln (1-\theta)} \frac{\theta^{n}}{n}, \quad n \geqslant 1
$$

Clearly, we have $p_{n}=C n^{-1} c^{n}$, where $c=\theta$ and $C=-1 / \ln (1-\theta)$.

When $\beta>1$, we use Stirling's formula $\Gamma(z) \sim(z / e)^{z} \sqrt{2 \pi / z}$, and after some tedious calculations we find that

$$
p_{n} \underset{n \rightarrow \infty}{\sim} \frac{(\beta /(\beta-1))^{1 / 2}}{\beta n \sqrt{2 \pi n}(-\log (1-\theta))}\left(\beta \theta\left(\frac{\beta(1-\theta)}{\beta-1}\right)^{\beta-1}\right)^{n} .
$$

Hence $p_{n}$ is of the form $p_{n}=c^{n} f_{n}$, where

$$
\begin{gathered}
c=c(\theta)=\beta \theta\left(\frac{\beta(1-\theta)}{\beta-1}\right)^{\beta-1} \\
f_{n} \sim \frac{(\beta /(\beta-1))^{1 / 2}}{n^{3 / 2} \beta \sqrt{2 \pi}(-\log (1-\theta))} \in \operatorname{RS}\left(-\frac{3}{2}\right) .
\end{gathered}
$$

Note that as a function of $\theta \in\left(0, \beta^{-1}\right), c(\theta)$ is increasing, and $c(\theta)<c\left(\beta^{-1}\right)=1$.

\section{Properties}

In this section, we study some basic properties of semi-heavy sequences and functions.

\subsection{Representation theorem}

From the theory of regular variation, we get the following representation theorem. An alternative representation theorem is stated by Albin and Sunden [1, Cor. 2.9]. 


\section{Theorem 1 [Representation theorem].}

(i) We have $\left(w_{n}\right) \in \operatorname{SHS}(c, \alpha)$ iff $w_{n}$ may be written as

$$
w_{n}=C_{n} c^{n} n^{\alpha} \exp \left(\sum_{i=a}^{n} \frac{\delta_{i}}{i}\right)
$$

where $C_{n} \rightarrow C \in(0, \infty)$ and $\delta_{n} \rightarrow 0$, and $a \geqslant 0$ is a constant.

(ii) We have $w \in \operatorname{SHF}(\beta, \alpha)$ iff $w(x)$ may be written as

$$
w(x)=C(x) x^{\alpha} \exp (-\beta x) \mathrm{L}(x),
$$

where $C(x) \rightarrow C>0$, and $\mathrm{L} \in \mathrm{RV}(0)$ satisfies $x \mathrm{~L}^{\prime}(x) / \mathrm{L}(x) \rightarrow 0$.

Proof. (i) This is Theorem 1.9.7 in Bingham et al. [4].

(ii) From the definition it follows that $w(x)=c(x) x^{\alpha} \exp (-\beta x) l(x)$, where $c(x) \rightarrow c>0$, and $l$ is slowly varying. From Bingham et al. [4, Thm. 1.3.3] (see also [4, Thm. 1.8.2]) we can find a slowly varying function $\mathrm{L}(x)$ such that $\mathrm{L}(x) \sim l(x)$ and $x \mathrm{~L}^{\prime}(x) / \mathrm{L}(x) \rightarrow 0$. It follows that $w(x)=C(x) x^{\alpha} \exp (-\beta x) \mathrm{L}(x)$, where $C(x)=c(x) l(x) / \mathrm{L}(x)$.

If $C(x)=C$ or if $C_{n}=C$ in the representation theorem, then we call the corresponding function (sequence) a normalized function (resp., sequence). For normalized functions and sequences, we have the following property.

\section{Proposition 1.}

(i) For normalized sequences, we have $\left(w_{n}\right) \in \operatorname{SHS}(c, \alpha)$ if and only if

$$
n\left(\frac{w_{n}}{w_{n-1}}-c\right) \rightarrow c \alpha .
$$

Moreover, (2.2) implies that $\left(w_{n-1}-w_{n}\right) \in \operatorname{SHS}(c, \alpha)$.

(ii) For normalized functions, we have $w \in \operatorname{SHF}(\beta, \alpha)$ if and only if

$$
x\left(\frac{w^{\prime}(x)}{w(x)}+\beta\right) \rightarrow \alpha .
$$

Moreover, (2.3) implies that $-w^{\prime}(x) \sim \beta w(x) \in \operatorname{SHF}(\beta, \alpha)$.

Proof. (i) For normalized sequences $\left(w_{n}\right) \in \operatorname{SHS}(c, \alpha)$ for large $n$, we have

$$
\frac{w_{n}}{w_{n-1}}-c=c\left(\frac{n^{\alpha}}{(n-1)^{\alpha}} \exp \frac{\delta_{n}}{n}-1\right) .
$$

Now we write

$$
\frac{n^{\alpha}}{(n-1)^{\alpha}} \exp \frac{\delta_{n}}{n}-1=\left(\frac{n^{\alpha}}{(n-1)^{\alpha}}-1\right) \exp \frac{\delta_{n}}{n}+\left(\exp \frac{\delta_{n}}{n}-1\right)
$$

Using

$$
\frac{n^{\alpha}}{(n-1)^{\alpha}}-1 \sim \alpha\left(\frac{n}{n-1}-1\right)=\frac{\alpha}{n-1}
$$


and $\exp \left(\delta_{n} / n\right)-1 \sim \delta_{n} / n$, we obtain (2.2). From $w_{n-1}-w_{n} \sim(1-c) w_{n-1}$ it also trivially follows that $\left(w_{n-1}-w_{n}\right) \in \operatorname{SHS}(c, \alpha)$.

For the converse, we assume that (2.2) holds, and we define $\left(f_{n}\right)$ by $f_{n}=c^{-n} w_{n}$. Using (2.2), it follows that

$$
n\left(\frac{f_{n}}{f_{n-1}}-1\right) \rightarrow \alpha
$$

and from here it follows that $\left(f_{n}\right) \in \operatorname{RS}(\alpha)$; see Bingham et al. [4, Thm. 1.9.8].

(ii) For a normalized function $w \in \operatorname{SHF}(\beta, \alpha)$, (2.1) with $C(x)=C$ shows that

$$
w^{\prime}(x)=\alpha \frac{w(x)}{x}-\beta w(x)+w(x) \frac{\mathrm{L}^{\prime}(x)}{\mathrm{L}(x)},
$$

and it follows that

$$
x\left(\frac{w^{\prime}(x)}{w(x)}+\beta\right)=\alpha+\frac{x \mathrm{~L}^{\prime}(x)}{\mathrm{L}(x)} \rightarrow \alpha .
$$

This proves (2.3). From here we obtain that $-w^{\prime}(x) \sim \beta w(x)$, so that $-w^{\prime}(x) \in \operatorname{SHF}(\beta, \alpha)$.

For the converse, (2.3) shows that $x\left(w^{\prime}(x) / w(x)+\beta\right)=\alpha(x)$, where $\alpha(x) \rightarrow \alpha$. It follows that $w^{\prime}(x) / w(x)=\alpha(x) / x-\beta$, and by integrating we see that

$$
\log w(x)=C-\beta x+\alpha \log x+\int_{a}^{x} \frac{\alpha(t)-\alpha}{t} \mathrm{~d} t .
$$

Hence $w \in \operatorname{SHF}(\beta, \alpha)$.

\section{Remarks.}

1. The examples $w(x)=[x]$ and $w_{n}=[e n]$ show that we need an extra condition to ensure that (2.3), resp., (2.2) holds.

2. If $w \in \operatorname{SHF}(\beta, \alpha)$, then the representation theorem shows that

$$
\log w(x)=\log C(x)-\beta x+\alpha \log x+\int_{a}^{x} \frac{\delta(u)}{u} \mathrm{~d} u .
$$

Since $\left(\log C(x)+\int_{a}^{x} u^{-1} \delta(u) \mathrm{d} u\right) / \log x \rightarrow 0$, we have

$$
\log w(x)=-\beta x+\alpha \log x+o(1) \log x .
$$

This opens a way to statistically estimate the important parameters $\alpha$ and $\beta$ and to see if it makes sense to model data by using a semi-heavy function.

\subsection{The "tail" of a semi-heavy function or sequence}

Inspired by Klüppelberg [12, p. 262], we prove the following Karamata-type result for the classes $\operatorname{LS}(c)$ and $\mathrm{L}(\beta)$.

\section{Proposition 2.}

(i) Let $c \in(0,1)$ and $t_{n}=\sum_{k=n}^{\infty} w_{k}<\infty$. We have $\left(w_{n}\right) \in \operatorname{LS}(c)$ if and only if $\left(t_{n}\right) \in \operatorname{LS}(c)$, and both statements imply that $t_{n} / w_{n} \rightarrow 1 /(1-c)$.

(ii) Let $\beta>0$ and $t(x)=\int_{x}^{\infty} w(z) \mathrm{d} z<\infty$. If $w \in \mathrm{L}(\beta)$, then $t \in \mathrm{L}(\beta)$ and $t(x) \sim w(x) / \beta$. Conversely, if $t \in \mathrm{L}(\beta)$ and if $w(x)$ is nonincreasing, then $w \in \mathrm{L}(\beta)$. 
Proof. (i) First, assume that $w_{n} / w_{n-1} \rightarrow c, 0<c<1$. It follows that, for $\varepsilon>0$ such that $0<c+\varepsilon<1$, we can find $n(\varepsilon)$ such that

$$
(c-\varepsilon) w_{n-1} \leqslant w_{n} \leqslant(c+\varepsilon) w_{n-1}, \quad n>n(\varepsilon) .
$$

For $k=0,1,2, \ldots$, we find that

$$
(c-\varepsilon)^{k} w_{n} \leqslant w_{n+k} \leqslant(c+\varepsilon)^{k} w_{n}, \quad n>n(\varepsilon) .
$$

Taking sums, we have

$$
\frac{w_{n}}{1-c+\varepsilon} \leqslant t_{n}=\sum_{i=n}^{\infty} w_{i} \leqslant \frac{w_{n}}{1-c-\varepsilon} .
$$

By letting $\varepsilon \rightarrow 0$ we conclude that $t_{n} / w_{n} \rightarrow 1 /(1-c)$, and it follows that $t_{n} / t_{n-1} \rightarrow c$.

To prove the converse, note that $c_{n-1}=t_{n-1}-t_{n}$. Using $t_{n} / t_{n-1} \rightarrow c$, we obtain that $c_{n-1} / t_{n-1} \rightarrow 1-c$, and hence the result.

(ii) Since $w(x+y) / w(x) \rightarrow \exp (-\beta y)$ for all $y$, we have $w(\log x) \in \mathrm{RV}(-\beta)$. Using $x^{-1} w(\log x) \in$ $\mathrm{RV}(-1-\beta)$, Karamata's theorem yields

$$
\frac{\int_{x}^{\infty} z^{-1} w(\log z) \mathrm{d} z}{w(\log x)} \rightarrow \frac{1}{\beta} .
$$

It follows that $t(\log x) \sim w(\log x) / \beta$. The result follows. To prove the converse, we take $y>0$. We have

$$
y w(x+y) \leqslant t(x)-t(x+y)=\int_{x}^{x+y} w(t) \mathrm{d} t \leqslant y w(x) .
$$

It follows that

$$
1-\mathrm{e}^{-\beta y} \leqslant \liminf \frac{y w(x)}{t(x)}
$$

and

$$
\limsup \frac{y w(x+y)}{t(x+y)} \leqslant \mathrm{e}^{\beta y}-1 .
$$

We obtain that

$$
\frac{1-\mathrm{e}^{-\beta y}}{y} \leqslant \lim \left(\begin{array}{c}
\sup \\
\inf
\end{array}\right) \frac{w(x)}{t(x)} \leqslant \frac{\mathrm{e}^{\beta y}-1}{y} .
$$

Since $y>0$ was arbitrary, we conclude that $w(x) / t(x) \rightarrow \beta .^{2}$ The result follows.

Remarks.

1. To pass from $t(\cdot)$ to $w(\cdot)$, in Proposition 2(ii), we assumed the Tauberian condition that $w$ is nonincreasing. Bingham et al. [4, Chap. 4] discuss more general Tauberian conditions.

2. If $w \in \operatorname{SHF}(\beta, \alpha), \beta>0$ and $\alpha<0$, then by Bingham et al. [4, Thm. 1.53] $f(x)=\mathrm{e}^{\beta x} w(x) \sim f_{0}(x)$, where $f_{0}(x) \downarrow 0$. We might assume that $f_{0}(x)$ is a tail distribution and study $w_{0}(x)=\mathrm{e}^{-\beta x} f_{0}(x)$ for tail distributions $f_{0}$. This is what has been done by Klüppelberg [12] and Xu et al. [20].

\footnotetext{
${ }^{2}$ The notation $a \leqslant \lim \left(\begin{array}{c}\sup \\ \text { inf }\end{array}\right) f(x) \leqslant b$ means that $a \leqslant \liminf f(x)$ and $\lim \sup f(x) \leqslant b$ both hold.
} 


\section{Corollary 1.}

(i) If $\left(w_{n}\right) \in \operatorname{SHS}(c, \alpha)$, then $\left(t_{n}\right) \in \operatorname{SHS}(c)$ and $t_{n} / w_{n} \rightarrow 1 /(1-c)$.

(ii) If $w \in \operatorname{SHF}(\beta, \alpha), \beta>0$, then $t \in \operatorname{SHF}(\beta, \alpha)$ and $t(x) \sim w(x) / \beta$.

\subsection{Convolutions}

Now consider two sequences $\left(u_{n}=c^{n} f_{n}\right) \in \operatorname{SHS}\left(c, \alpha_{u}\right)$ and $\left(v_{n}=c^{n} g_{n}\right) \in \operatorname{SHS}\left(c, \alpha_{v}\right)$ and consider the convolution $(u * v)_{n}=\sum_{k=0}^{n} u_{k} v_{n-k}$. Using the definition, we get that $(u * v)_{n}=c^{n}(f * g)_{n}$. In the case of functions, in a similar way, we have $u * v(x)=\mathrm{e}^{-\beta x} f * g(x)$, where $f * g(x)=\int_{0}^{x} f(y) g(x-y) \mathrm{d} y$.

In the next subsections, we consider two cases. In the first case, we assume that $\left(u_{n}=c^{n} f_{n}\right) \in \operatorname{SHS}\left(c, \alpha_{u}\right)$ and $\sum_{k=0}^{\infty} f_{k}<\infty$. For functions, we consider the case where $u(x)=\mathrm{e}^{-\beta x} f(x)$ and $\int_{0}^{\infty} f(x) \mathrm{d} x<\infty$. In the second case, we assume that the sum or the integral is not finite.

\subsubsection{Finite sums or finite integrals}

We have the following result inspired by Chover et al. [6].

\section{Proposition 3.}

(i) Suppose that $\left(u_{n}\right) \in \operatorname{SHS}\left(c, \alpha_{u}\right)$ and $\left(v_{n}\right) \in \operatorname{SHS}\left(c, \alpha_{v}\right)$. Let $f_{n}=c^{-n} u_{n}$ and $g_{n}=c^{-n} v_{n}$ and assume that $F=\sum_{n=0}^{\infty} f_{n}<\infty$ and $G=\sum_{n=0}^{\infty} g_{n}<\infty$. Then $(u * v)_{n}=F v_{n}+G u_{n}+o\left(u_{n}\right)+o\left(v_{n}\right)$ and $\left(u^{* 2}\right)_{n} \sim 2 F u_{n}$.

(ii) Suppose that $u \in \operatorname{SHF}(\beta, \alpha)$ and $v \in \operatorname{SHF}(\beta, \beta)$. Let $f(x)=\mathrm{e}^{\beta x} u(x)$ and $g(x)=\mathrm{e}^{\beta x} v(x)$ and assume that $F=\int_{0}^{\infty} f(t) \mathrm{d} t<\infty$ and $G=\int_{0}^{\infty} g(t) \mathrm{d} t<\infty$. Then $u * v(x)=F v(x)+G u(x)+$ $o(u(x))+o(v(x))$ and $u^{* 2}(x) \sim 2 F u(x)$.

Proof. For convenience, we prove (ii). We have $u * v(x)=\mathrm{e}^{-\beta x} f * g(x)$ and

$$
f * g(x)=\int_{0}^{x / 2} f(t) g(x-t) \mathrm{d} t+\int_{0}^{x / 2} g(t) f(x-t) \mathrm{d} t=\mathrm{I}+\mathrm{II} .
$$

Using the local uniform convergence, we obtain that $\lim _{x \rightarrow \infty} \mathrm{I} / g(x)=F$ and $\lim _{x \rightarrow \infty} \mathrm{II} / f(x)=G$. It follows that $f * g(x)=F g(x)+G f(x)+o(g(x))+o(f(x))$, and the first result follows. In the particular case $u=v$, we find that $u * u(x) \sim 2 F u(x)$.

(i) The proof is similar.

\section{Remarks.}

1. Under the conditions of Proposition 3(i), we have $(u * v)_{n}=c^{n}(f * g)_{n}$ and $\sum_{n=0}^{\infty}(f * g)_{n}=F+G$.

2. Under the conditions of Proposition 3(i), we have $(u * u)_{n} \sim 2 F u_{n}$ and hence also that $\left(\left(u^{* 2}\right)_{n}\right) \in$ $\operatorname{SHS}(c, \alpha)$ satisfies the summability condition of Proposition 3(i).

Recall that a sequence $\left(f_{n}\right)$ is in the class of subexponential sequences if $f_{n}>0,\left(f_{n}\right) \in \operatorname{LS}, \sum_{k=0}^{\infty} f_{k}<\infty$, and $f_{n}^{* 2} / f_{n} \rightarrow 2 \sum_{k=0}^{\infty} f_{k}$. Notation: $\left(f_{n}\right) \in$ SS. In a similar way, we say that a positive function $f(x)$ is in the class of subexponential functions if $f(x) \in \mathrm{L} \cap \mathrm{L}_{1}([0, \infty))$ and if $f^{* 2}(x) / f(x) \rightarrow 2 \int_{0}^{\infty} f(t) \mathrm{d} t$. Notation: $f \in \mathrm{SF}$. It is well known that if $\left(f_{n}\right) \in \operatorname{RS}(\alpha), \alpha \leqslant-1$, and (in the case $\left.\alpha=-1\right) \sum_{k=0}^{\infty} f_{k}<\infty$, then $\left(f_{n}\right) \in \mathrm{SS}$. A survey of applications of SS and SF in probability theory can be found in Bingham et al. [4, Appendix 4] or [9]. In the discrete case, see also Embrechts and Omey [8].

In the next result, we consider $k$-fold convolutions. 


\section{Proposition 4.}

(i) Suppose that $\left(u_{n}\right) \in \operatorname{SHS}(c, \alpha)$. Let $f_{n}=c^{-n} u_{n}$ and assume that $F=\sum_{n=0}^{\infty} f_{n}<\infty$. Then $\left(\left(u^{* k}\right)_{n}\right) \in \operatorname{SHS}(c, \alpha)$ and $\left(u^{* k}\right)_{n} \sim k F^{k-1} u_{n}$. Moreover, for each $\epsilon>0$, we can find a constant $K>0$ such that

$$
\left(u^{* k}\right)_{n} \leqslant K F^{k-1}(1+\epsilon)^{k} u_{n}, \quad k \geqslant 1, n \geqslant 1 .
$$

(ii) Suppose that $u \in \operatorname{SHF}(\beta, \alpha)$. Let $f(x)=\mathrm{e}^{\beta x} u(x)$ and assume that $F=\int_{0}^{\infty} f(t) \mathrm{d} t<\infty$. Then $u^{* k} \in \operatorname{SHF}(\beta, \alpha)$ and $u^{* k}(x) \sim k F^{k-1} u(x)$. Moreover, for each $\epsilon>0$, we can find a constant $K>0$ such that

$$
u^{* k}(x) \leqslant K F^{k-1}(1+\epsilon)^{k} u(x), \quad k \geqslant 1, x \geqslant 0 .
$$

Proof. (i) The proof of the first part follows from Proposition 3, and the inequality follows from the results of Chover et al. [6]; see also Klüppelberg [12, Lemma 3.1].

(ii) Proof is similar.

\section{Remarks.}

1. When $\alpha<-1$, the summability condition, resp., integral condition is automatically satisfied. Only in the case $\alpha=-1$, we need to assume this.

2. If $w \in \operatorname{SHF}(\beta, \alpha), \beta>0$, then Corollary 1 shows that $t \in \operatorname{SHF}(\beta, \alpha)$ and $t(x) \sim w(x) / \beta$. It follows from Proposition 4 that $t^{* 2}(x) \sim 2 t(x) \int_{0}^{\infty} f(s) \mathrm{d} s$.

\subsubsection{Infinite sums or infinite integrals}

Now we consider the case where $w_{n}=c^{n} f_{n}$ with $\left(f_{n}\right) \in \operatorname{RS}(\alpha), \alpha \geqslant-1$, such that $\sum_{k=0}^{\infty} f_{k}=\infty$. The following result can easily be reformulated for functions.

Theorem 2. Assume that $\left(u_{n}\right) \in \operatorname{SHS}(c, \alpha)$ and $\left(v_{n}\right) \in \operatorname{SHS}(c, \beta)=c^{n} f_{n}$ and let $f_{n}=c^{-n} u_{n}, g_{n}=c^{-n} v_{n}$. Assume that $\left(f_{n}\right) \in \operatorname{RS}(\alpha), \alpha \geqslant-1,\left(g_{n}\right) \in \operatorname{RS}(\beta), \beta \geqslant-1$. If $\alpha=-1$, resp., $\beta=-1$, then assume also that $\sum_{k=0}^{n} f_{k} \rightarrow \infty$, resp., $\sum_{k=0}^{n} g_{k} \rightarrow \infty$.

(i) If $\alpha>-1$ and $\beta>-1$, then

$$
\frac{(u * v)_{n}}{c^{n} n f_{n} g_{n}} \rightarrow \mathrm{B}(\alpha+1, \beta+1),
$$

where $\mathrm{B}(\cdot, \cdot)$ denotes the beta-function. If $\alpha=-1$ and $\beta>-1$, then

$$
\frac{(u * v)_{n}}{c^{n} g_{n} \sum_{k=0}^{n} f_{k}} \rightarrow 1
$$

(ii) If $\alpha>-1$ and $\beta=-1$, then

$$
\frac{(u * v)_{n}}{c^{n} f_{n} \sum_{k=0}^{n} g_{k}} \rightarrow 1
$$

(iii) If $\alpha=\beta=-1$, then

$$
\frac{(u * v)_{n}}{c^{n}\left(g_{n} \sum_{k=0}^{n} f_{k}+f_{n} \sum_{k=0}^{n} g_{k}\right)} \rightarrow 1
$$


Proof. We fix $\epsilon, 0<\epsilon<1 / 2$, and write

$$
\frac{1}{c^{n}}(u * v)_{n}=\left(\sum_{k=0}^{[n \epsilon]-1}+\sum_{k=[n \epsilon]}^{n-[n \epsilon]}+\sum_{k=n-[n \epsilon]+1}^{n}\right) f_{k} g_{n-k}=: \mathrm{I}+\mathrm{II}+\mathrm{III} .
$$

First, we consider II. Note that $\int_{k \leqslant x<k+1} f_{[x]} g_{n-[x]} \mathrm{d} x=f_{k} g_{n-k}$. Taking sums, we find that

$$
\mathrm{II}=\int_{[n \epsilon] \leqslant x<n-[n \epsilon]+1} f_{[x]} g_{n-[x]} \mathrm{d} x .
$$

Replacing $x$ by $n t$, we have

$$
\mathrm{II}=n \int_{[n \epsilon] / n}^{1-[n \epsilon] / n+1 / n} f_{[n t]} g_{n-[n t]} \mathrm{d} t .
$$

Using the local uniform convergence, we obtain that

$$
\lim _{n \rightarrow \infty} \frac{\mathrm{II}}{n f_{n} g_{n}}=\int_{\epsilon}^{1-\epsilon} t^{\alpha}(1-t)^{\beta} \mathrm{d} t .
$$

Note that (2.4) holds for $\alpha \geqslant-1$ an $\beta \geqslant-1$.

Next, we consider I and III. We have

$$
\frac{\mathrm{I}}{g_{n}}=\sum_{k=0}^{[n \epsilon]-1} f_{k} \frac{g_{n-k}}{g_{n}} ; \quad \frac{\mathrm{III}}{f_{n}}=\sum_{k=0}^{[n \epsilon]-1} g_{k} \frac{f_{n-k}}{f_{n}} .
$$

We consider several cases.

a) First, consider the case where $\alpha>-1$ and $\beta>-1$. We have

$$
\frac{\mathrm{I}}{g_{n}} \leqslant \sup _{0 \leqslant k \leqslant[n \epsilon]-1} \frac{g_{n-k}}{g_{n}} \sum_{j=0}^{[n \epsilon]-1} f_{j} .
$$

If $\alpha>-1$, then Karamata's theorem [4, Thm. 1.5.8] for sequences gives that

$$
\sum_{j=0}^{[n \epsilon]-1} f_{j} \sim \frac{1}{1+\alpha}[n \epsilon] f_{[n \epsilon]} \sim \frac{\epsilon^{1+\alpha}}{1+\alpha} n f_{n} .
$$

On the other hand, we have

$$
\sup _{0 \leqslant k \leqslant[n \epsilon]-1} \frac{g_{n-k}}{g_{n}} \leqslant \sup _{0 \leqslant k \leqslant n / 2} \frac{g_{n-k}}{g_{n}}
$$

which is bounded as $n \rightarrow \infty$. We conclude that

$$
\limsup \frac{\mathrm{I}}{n f_{n} g_{n}} \leqslant B \frac{\epsilon^{1+\alpha}}{1+\alpha},
$$


where the constant $B$ does not depend on $\epsilon$. In a similar way, if $\beta>-1$, then we find that

$$
\limsup \frac{\mathrm{III}}{n f_{n} g_{n}} \leqslant B^{\prime} \frac{\epsilon^{1+\beta}}{1+\beta}
$$

for some constant $B^{\prime}$ that does not depend on $\epsilon$.

Now, combining (2.4), (2.6), and (2.7), we obtain that

$$
\int_{\epsilon}^{1-\epsilon} t^{\alpha}(1-t)^{\beta} \mathrm{d} t \leqslant \lim _{n \rightarrow \infty}\left(\begin{array}{c}
\sup \\
\text { inf }
\end{array}\right) \frac{\mathrm{I}+\mathrm{II}+\mathrm{III}}{n f_{n} g_{n}} \leqslant \int_{\epsilon}^{1-\epsilon} t^{\alpha}(1-t)^{\beta} \mathrm{d} t+B \frac{\epsilon^{1+\alpha}}{1+\alpha}+B^{\prime} \frac{\epsilon^{1+\beta}}{1+\beta} .
$$

Now let $\epsilon \rightarrow 0$ to obtain the first result.

b) Now consider the case $\alpha=-1, \beta>-1$. In the case of $\alpha=-1$, Karamata's theorem [4, Prop. 1.5.9a] shows that we have $\left(\sum_{k=0}^{n} f_{k}\right) \in \operatorname{RS}(0)$ and $n f_{n}=o(1) \sum_{k=0}^{n} f_{k}$. In view of (2.4) and (2.7), it follows that

$$
\lim _{n \rightarrow \infty} \frac{\mathrm{II}}{g_{n} \sum_{k=0}^{n} f_{k}}=\lim _{n \rightarrow \infty} \frac{\mathrm{III}}{g_{n} \sum_{k=0}^{n} f_{k}}=0 .
$$

Now we reconsider I, and to this end, we have to study $g_{n-k} / g_{n}$ for $0 \leqslant k \leqslant[n \epsilon]-1$. Using the local uniform convergence theorem $[4$, Thm. 1.5.2] again, we have

$$
\sup _{0 \leqslant k \leqslant[n \epsilon]-1} \frac{g_{n-k}}{g_{n}}=\sup _{1-\epsilon \leqslant t \leqslant 1} \frac{g_{[n t]}}{g_{n}} \rightarrow \sup _{1-\epsilon \leqslant t \leqslant 1} t^{\beta}
$$

and similarly

$$
\inf _{0 \leqslant k \leqslant[n \epsilon]-1} \frac{g_{n-k}}{g_{n}} \rightarrow \inf _{1-\epsilon \leqslant t \leqslant 1} t^{\beta}
$$

This implies that there is an integer $n_{0}=n_{0}(g, \epsilon)$ so that, for all $n \geqslant n_{0}$,

$$
(1-\epsilon) \min \left(1,(1-\epsilon)^{\beta}\right) \leqslant \frac{g_{n-k}}{g_{n}} \leqslant(1+\epsilon) \max \left(1,(1-\epsilon)^{\beta}\right),
$$

uniformly in $k=0,1, \ldots,[n \epsilon]$. In the place of $(2.5)$, we find that

$$
(1-\epsilon) \min \left(1,(1-\epsilon)^{\beta}\right) \sum_{k=0}^{[n \epsilon]-1} f_{k} \leqslant \frac{\mathrm{I}}{g_{n}} \leqslant(1+\epsilon) \max \left(1,(1-\epsilon)^{\beta}\right) \sum_{k=0}^{[n \epsilon]-1} f_{k}, \quad n \geqslant n_{0} .
$$

Since $\left(\sum_{k=0}^{n} f_{k}\right) \in \operatorname{RS}(0)$, it follows that

$$
(1-\epsilon) \min \left(1,(1-\epsilon)^{\beta}\right) \leqslant \lim \left(\begin{array}{c}
\sup \\
\inf
\end{array}\right) \frac{\mathrm{I}}{g_{n} \sum_{k=0}^{n} f_{k}} \leqslant(1+\epsilon) \max \left(1,(1-\epsilon)^{\beta}\right) .
$$

Combining this with (2.8), we conclude that

$$
(1-\epsilon) \min \left(1,(1-\epsilon)^{\beta}\right) \leqslant \lim \left(\begin{array}{c}
\sup \\
\inf
\end{array}\right) \frac{\mathrm{I}+\mathrm{II}+\mathrm{III}}{g_{n} \sum_{k=0}^{n} f_{k}} \leqslant(1+\epsilon) \max \left(1,(1-\epsilon)^{\beta}\right) .
$$


Now let $\epsilon \rightarrow 0$ to find that

$$
\lim \frac{\mathrm{I}+\mathrm{II}+\mathrm{III}}{g_{n} \sum_{k=0}^{n} f_{k}}=1 .
$$

c) The case of $\beta=-1$ and $\alpha>-1$ can be treated in a similar way.

d) Now consider the case where $\alpha=\beta=-1$. Using a similar approach as before, now we get that

$$
\lim _{n \rightarrow \infty} \frac{\mathrm{II}}{g_{n} \sum_{k=0}^{n} f_{k}+f_{n} \sum_{k=0}^{n} g_{k}}=0
$$

and

$$
\lim _{n \rightarrow \infty} \frac{\mathrm{I}+\mathrm{III}}{g_{n} \sum_{k=0}^{n} f_{k}+f_{n} \sum_{k=0}^{n} g_{k}}=1 .
$$

This proves the theorem.

Remark. If $\alpha \geqslant-1$ and $\beta \geqslant-1$, then $(u * v)_{n}$ is again of the form $(u * v)_{n}=C c^{n} h_{n}$, and $\left(h_{n}\right) \in$ $\operatorname{RS}(1+\alpha+\beta)$.

In the particular case where $u_{n}=v_{n}$, we get the following corollary. We also formulate it for functions.

\section{Corollary 2.}

(i) Assume that $u_{n}=c^{n} f_{n}$, where $0<c<1$ and $\left(f_{n}\right) \in \operatorname{RS}(\alpha)$.

(a) If $\alpha>-1$, then

$$
\frac{\left(u^{* 2}\right)_{n}}{n c^{n} f_{n}^{2}}=\frac{\left(u^{* 2}\right)_{n}}{u_{n} n f_{n}} \rightarrow \mathrm{B}(1+\alpha, 1+\alpha) .
$$

(b) If $\alpha=-1$ and $\sum_{i=0}^{n} f_{i} \rightarrow \infty$, then

$$
\frac{\left(u^{* 2}\right)_{n}}{c^{n} f(n) \sum_{i=0}^{n} f_{i}}=\frac{\left(u^{* 2}\right)_{n}}{u_{n} \sum_{i=0}^{n} f_{i}} \rightarrow 2 .
$$

(ii) Assume that $w(x)=\mathrm{e}^{-\beta x} f(x), \beta>0$, and $f \in \operatorname{RV}(\alpha)$.

(a) If $\alpha>-1$, then

$$
\frac{w^{* 2}(x)}{x e^{-\beta x} f^{2}(x)}=\frac{w^{* 2}(x)}{w(x) x f(x)} \rightarrow \mathrm{B}(1+\alpha, 1+\alpha) .
$$

(b) If $\alpha=-1$ and $\int_{0}^{x} f(t) \mathrm{d} t \rightarrow \infty$, then

$$
\frac{w^{* 2}(x)}{\mathrm{e}^{-\beta x} f(x) \int_{0}^{x} f(t) \mathrm{d} t}=\frac{w^{* 2}(x)}{w(x) \int_{0}^{x} f(t) \mathrm{d} t} \rightarrow 2 .
$$

For higher-order convolutions, we have the following result. A similar result holds for functions.

Theorem 3. Assume that $u_{n}=c^{n} f_{n}$, where $0<c<1$ and $\left(f_{n}\right) \in \operatorname{RS}(\alpha)$. For all $k \geqslant 2$, we have:

(i) If $\alpha>-1$, then

$$
\frac{\left(u^{* k}\right)_{n}}{c^{n} n^{k-1} f_{n}^{k}} \rightarrow \prod_{i=2}^{k} \mathrm{~B}(1+\alpha,(i-1)(1+\alpha)) .
$$


(ii) If $\alpha=-1$ and $\sum_{i=0}^{\infty} f_{i}=\infty$, then

$$
\frac{\left(u^{* k}\right)_{n}}{c^{n} f_{n}\left(\sum_{i=0}^{n} f_{i}\right)^{k-1}} \rightarrow k !
$$

Proof. (i) We use induction. The result holds for $k=2$, and we have $\left(u^{* 2}\right)_{n}=c^{n} f_{2}(n)$, where $f_{2}(n) \sim$ $n f_{n}^{2} \mathrm{~B}(1+\alpha, 1+\alpha) \in \operatorname{RS}(1+2 \alpha)$. Using the theorem (with $u_{n}$ and $\left.\left(u^{* 2}\right)_{n}\right)$, we obtain that

$$
\frac{\left(u^{* 3}\right)_{n}}{c^{n} n f_{n} f_{2}(n)} \rightarrow \mathrm{B}(1+\alpha, 2+2 \alpha)
$$

and the result follows for $k=3$. Now the result easily follows by induction.

(ii) For $\alpha=-1$ and $k=2$, we have proved that $\left(u^{* 2}\right)_{n}=c^{n} f_{2}(n)$, where $f_{2}(n)=2 f_{n} h_{1}(n)$ and $h_{1}(n)=\sum_{k=0}^{n} f_{k}$. Note that $\left(f_{2}(n)\right) \in \operatorname{RS}(-1)$. Using the theorem (with $u_{n}$ and $\left.\left(u^{* 2}\right)_{n}\right)$, we obtain that $\left(u^{* 3}\right)_{n}=c^{n} f_{3}(n)$, where

$$
f_{3}(n)=f_{2}(n) h_{1}(n)+f_{n} \sum_{k=0}^{n} f_{2}(k) .
$$

Note that $f_{3}(n)=2 f_{n}\left(h_{2}(n)+g(n)\right)$, where $g(n)=\sum_{k=0}^{n} f_{k} \sum_{i=0}^{k} f_{i}$ and $h_{2}(n)=\left(\sum_{k=0}^{n} f_{k}\right)^{2}$. We have

$$
\begin{aligned}
g(n+1)-g(n) & =f_{n+1} \sum_{i=0}^{n+1} f_{i} \sim f_{n} \sum_{k=0}^{n} f_{k}, \\
h_{2}(n+1)-h_{2}(n) & =f_{n+1}\left(\sum_{k=0}^{n+1} f_{k}+\sum_{k=0}^{n} f_{k}\right) \sim 2 f_{n} \sum_{k=0}^{n} f_{k},
\end{aligned}
$$

and we obtain that $h_{2}(n+1)-h_{2}(n) \sim 2(g(n+1)-g(n))$ as $n \rightarrow \infty$. For $n \geqslant n^{\circ}$ and $0<\epsilon<1$, we get that

$$
(1-\epsilon) 2(g(n+1)-g(n)) \leqslant h_{2}(n+1)-h_{2}(n) \leqslant(1+\epsilon) 2(g(n+1)-g(n)), \quad n \geqslant n^{\circ} .
$$

Taking sums $\sum_{n=m}^{K}$, for fixed $m$, we get

$$
(1-\epsilon) 2(g(K+1)-g(m)) \leqslant h_{2}(K+1)-h_{2}(m) \leqslant(1+\epsilon) 2(g(K+1)-g(m)), \quad K \geqslant m \geqslant n^{\circ} .
$$

Since $h(K), g(K) \rightarrow \infty$ as $K \rightarrow \infty$, we obtain that

$$
2(1-\epsilon) \leqslant \lim \left(\begin{array}{c}
\sup \\
\inf
\end{array}\right) \frac{h_{2}(K+1)}{g(K+1)} \leqslant 2(1+\epsilon)
$$

Since $\epsilon>0$ was arbitrary, we conclude that $h_{2}(n) \sim 2 g(n)$. It follows that $f_{3}(n) \sim 3 ! f(n) h_{2}(n) \in \operatorname{RS}(-1)$.

Now assume that $\left(u^{* r}\right)_{n} \sim c^{n} f_{r}(n)$ where $f_{r}(n) \sim r ! f(n) h_{r-1}(n) \in \operatorname{RS}(-1)$ with $h_{r-1}(n)=$ $\left(\sum_{i=0}^{n} f(i)\right)^{r-1}$. Using the theorem with $u_{n}$ and $\left(u^{* r}\right)_{n}$, we obtain that $\left(u^{*(r+1)}\right)_{n} \sim c^{n} f_{r+1}(n)$, where

$$
\left.\left.f_{r+1}(n)=r ! f_{n} h_{r-1}(n) \sum_{k=1}^{n} f_{k}+r ! f_{n} \sum_{k=0}^{n} f_{k} h_{r-1}(k)\right)=r ! f_{n}\left(h_{r}(n)+g(n)\right)\right) \text {. }
$$


where $g^{r}(n)=\sum_{k=0}^{n} f_{k} h_{r-1}(k)$. Clearly, we have $h_{r}(n+1)-h_{r}(n) \sim f_{n} r h_{r-1}(n)$. For $g^{r}(n)$, we have $g^{r}(n+1)-g^{r}(n) \sim f_{n} h_{r-1}(n)$. As before, we find that $h_{r}(n) \sim r g^{r}(n)$, and we conclude that $f_{r+1}(n) \sim$ $(r+1) ! f_{n} h_{r}(n)$. This proves the result.

Remark. From the result it follows that:

(i) for $\alpha>-1$,

$$
\frac{\left(u^{* k}\right)_{n}}{u_{n}} \sim n^{k-1} f_{n}^{k-1} \prod_{i=2}^{k} \mathrm{~B}(1+\alpha,(i-1)(1+\alpha)) \in \operatorname{RS}((k-1)(1+\alpha)) .
$$

(ii) for $\alpha=-1$,

$$
\frac{u_{n}^{* k}}{u_{n}} \sim k !\left(\sum_{i=0}^{n} f_{i}\right)^{k-1} \in \operatorname{RS}(0) .
$$

It may be interesting to study general sequences that satisfy $\left(\left(u^{* 2}\right)_{n} / u_{n}\right) \in \operatorname{RS}(\theta)$ or $\left(\left(u^{* k}\right)_{n} / u_{n}\right) \in$ $\operatorname{RS}((k-1) \theta)$.

\subsubsection{Examples}

Our results can be applied for the logistic model where $u_{n}=1 /\left(1+\mathrm{e}^{n}\right)$. Here we have $u_{n}=f_{n} \mathrm{e}^{-n}$, where $f_{n} \rightarrow 1$. Theorem 3 (with $\alpha=0$ ) shows that

$$
\frac{\left(u^{* k}\right)_{n}}{\mathrm{e}^{-n} n^{k-1}} \rightarrow \prod_{i=2}^{k} \mathrm{~B}(1, i-1)
$$

Using $\mathrm{B}(x, y)=\Gamma(x) \Gamma(y) / \Gamma(x+y)$, we have $\mathrm{B}(1, i-1)=\Gamma(i-1) / \Gamma(i)=1 /(i-1)$, and we find that

$$
\frac{\left(u^{* k}\right)_{n}}{\mathrm{e}^{-n} n^{k-1}} \rightarrow \frac{1}{(k-1) !}
$$

In the case where $w_{n}=(n+1)^{\alpha} \mathrm{e}^{-\beta n}, n \geqslant 0$, we have $w_{n}=c^{n} f_{n}$ where $c=\mathrm{e}^{-\beta}$ and $\left(f_{n}=(n+1)^{\alpha}\right) \in$ $\operatorname{RS}(\alpha)$. For the $k$-fold convolution, we obtain the following results:

(i) If $\alpha>-1$, then

$$
\frac{\left(w^{* k}\right)_{n}}{\mathrm{e}^{-\beta n} n^{\alpha k+k-1}} \rightarrow \prod_{i=2}^{k} \mathrm{~B}(1+\alpha,(i-1)(1+\alpha)) .
$$

(ii) If $\alpha=-1$, then $\sum_{i=0}^{n} f(i) \sim \log (n)$ and

$$
\frac{n\left(w^{* k}\right)_{n}}{c^{n}(\log (n))^{k-1}} \rightarrow k !
$$

Now suppose that $X$ is a r.v. with d.f. $F(x)=\mathbf{P}(X \leqslant x)$, tail $\bar{F}(x)=1-F(x)$, and density $f$. Define $F \times G(x)=\int_{0}^{x} F(x-y) \mathrm{d} G(x)$, so that $F^{\times k}=F^{\times(k-1)} \times F$ and $\overline{F^{\times k}}=1-F^{\times k}$. We have the following result.

Proposition 5. Suppose that $f \in \operatorname{SHF}(\beta, \alpha)$ and let $g(x)=\mathrm{e}^{\beta x} f(x)$. Assume that $g \in \operatorname{RV}(\alpha)$.

(i) If $\alpha>-1$, then $\overline{F^{\times 2}}(x) \sim \bar{F}(x) x g(x) \mathrm{B}(1+\alpha, 1+\alpha)$.

(ii) If $\alpha=-1$, then $\overline{F^{\times 2}}(x) \sim 2 \bar{F}(x) \int_{0}^{x} g(t) \mathrm{d} t$. 
Proof. (i) Assume that $f(x)=\mathrm{e}^{-\beta x} g(x) \in \mathrm{L}(\beta)$. Using Proposition 2, we have $\bar{F}(x) \sim f(x) / \beta$. Corollary 2 gives that $f^{* 2}(x) \sim x f(x) g(x) \mathrm{B}(1+\alpha, 1+\alpha) \in \operatorname{SHF}(\beta, 2 \alpha+1)$. Taking integrals and using Proposition 2, it follows that

$$
\overline{F^{\times 2}}(x) \sim f(x) x g(x) \frac{\mathrm{B}(1+\alpha, 1+\alpha)}{\beta} \sim \bar{F}(x) x g(x) \mathrm{B}(1+\alpha, 1+\alpha) .
$$

(ii) Again we have $\bar{F}(x) \sim f(x) / \beta$. Now Corollary 2 gives that $f^{* 2}(x) \sim 2 f(x) \int_{0}^{x} g(t) \mathrm{d} t \in \mathrm{SHF}(\beta,-1)$. Taking integrals and using Proposition 2 , it follows that

$$
\overline{F^{\times 2}}(x) \sim \frac{2 f(x) \int_{0}^{x} g(t) \mathrm{d} t}{\beta} \sim 2 \bar{F}(x) \int_{0}^{x} g(t) \mathrm{d} t .
$$

In the case of $k>2$ and $\alpha>-1$, Corollary 2 gives that $f^{* k}(x) \sim x^{k-1} f(x) g^{k-1}(x) C(\alpha, k) \in \mathrm{L}(\beta)$. Taking integrals and using Proposition 2, we obtain that

$$
\overline{F^{\times k}}(x) \sim \frac{1}{\beta} C(\alpha, k) x^{k-1} f(x) g^{k-1}(x) \sim C(\alpha, k) \bar{F}(x) x^{k-1} g^{k-1}(x) .
$$

In the case of $\alpha=-1$, we obtain that

$$
\overline{F^{\times k}}(x) \sim k ! \bar{F}(x)\left(\int_{0}^{x} g(t) \mathrm{d} t\right)^{k-1} .
$$

Remark. In the usual subexponential case we have that $\overline{F^{\times 2}}(x) \sim 2 \bar{F}(x)$. Our results show that it makes sense to study tails for which $\overline{F^{\times 2}}(x) / \bar{F}(x) \rightarrow \infty$. Our conditions give a result of this type together with the rate at which $\overline{F^{\times 2}}(x) / \bar{F}(x)$ tends to infinity. See Schmidli [17] for a different approach.

\subsubsection{An upper bound without regular variation}

In the next result we do not use regular variation to obtain an upper bound for $\left(u^{* k}\right)_{n}$ when $u_{n}=c^{n} f_{n}$, $0<c<1$.

\section{Lemma 1.}

(i) Let $h_{n}=\sum_{i=0}^{n} f_{i}^{2}$ and $u_{n}=c^{n} f_{n}$. For all $k \geqslant 2$, we have $\left(u^{* k}\right)_{n} \leqslant c^{n} n^{(k-2) / 2} h_{n}^{k / 2}$.

(ii) Let $h(x)=\int_{0}^{x} f^{2}(t) \mathrm{d}$ t and $w(x)=\mathrm{e}^{-\beta x} f(x)$. For all $k \geqslant 2$, we have $w^{* k}(x) \leqslant \mathrm{e}^{-\beta x} x^{(k-2) / 2} h^{k / 2}(x)$.

Proof. (i) First, consider $k=2$. Using the Cauchy-Schwarz inequality, we have

$$
(u * u)_{n}=c^{n} \sum_{j=0}^{n} f_{j} f_{n-j} \leqslant c^{n} \sqrt{\sum_{j=0}^{n}\left(f^{2}\right)_{j} \times \sum_{j=0}^{n}\left(f^{2}\right)_{j}}=c^{n} h_{n} .
$$

This is the inequality for $k=2$. Assuming that the formula holds for $k=2,3, \ldots, m$, we consider $\left(u^{*(m+1)}\right)_{n}=$ $\left(u^{* m} * u\right)_{n}$, and we find

$$
\left(u^{*(m+1)}\right)_{n}=\sum_{j=0}^{n} c^{j} f_{j}\left(u^{* m}\right)_{n-j} \leqslant c^{n} \sum_{j=0}^{n}(n-j)^{(m-2) / 2} f_{j} h_{n-j}^{m / 2} .
$$


Now the Cauchy-Schwarz inequality leads to

$$
\left(u^{*(m+1)}\right)_{n} \leqslant c^{n} \sqrt{h_{n} \sum_{j=0}^{n} j^{(m-2)} h_{j}^{m}} .
$$

Since $h_{j}$ is nondecreasing and since $j \leqslant n$, we find that

$$
\left(u^{*(m+1)}\right)_{n} \leqslant c^{n} \sqrt{h_{n} n n^{(m-2)} h_{n}^{m}},
$$

and we find that $\left(u^{*(m+1)}\right)_{n} \leqslant c^{n} n^{(m-1) / 2} h_{n}^{(m+1) / 2}$. This proves the result.

(ii) The proof of (ii) is similar.

Remarks.

1. If $\left(f_{n}\right) \in \operatorname{RS}(\alpha)$ with $2 \alpha>-1$, then $h_{n} \sim n f_{n}^{2} /(2 \alpha+1)$, and we find that

$$
\frac{\left(u^{* 2}\right)_{n}}{c_{n} n f_{n}^{2}} \leqslant \frac{h_{n}}{n f_{n}^{2}}=O(1), \quad \frac{\left(u^{* k}\right)_{n}}{c^{n} n^{k-1} f_{n}^{k}} \leqslant \frac{n^{(k-2) / 2} h_{n}^{k / 2}}{n^{k-1} f_{n}^{k}}=O(1) .
$$

2. If $f \in \operatorname{RV}(\alpha)$ with $2 \alpha>-1$, then we have $h(x) \sim x f^{2}(x) /(1+2 \alpha)$,

$$
x^{(k-2) / 2}(h(x))^{k / 2} \sim(1+2 \alpha)^{-k / 2} x^{k-1} f^{k}(x),
$$

and $w^{* k}(x)=O(1) w(x)(x f(x))^{k-1}$.

\section{Applications}

\subsection{Subordination}

Let $N$ denote a r.v. with $\mathbf{P}(N=k)=p_{k}, k \geqslant 1$, and let $X$ denote a r.v. with density $w(x)$. Suppose that $N$ and $X$ are independent. If $X, X_{1}, X_{2}, \ldots$ are i.i.d., then for each $n \geqslant 1$, the sum $S_{n}=\sum_{i=1}^{n} X_{i}$ has the density $w^{* n}(x)$. The random sum $S_{N}$ has the density $g(x)=\sum_{n=1}^{\infty} p_{n} w^{* n}(x)$. Inspired by Schmidli [17], we prove the following result.

Theorem 4. Suppose that $\left(p_{n}\right) \in \operatorname{SS}$ and $w \in \operatorname{SHF}(\beta, \alpha)$. Let $h(x)=\mathrm{e}^{\beta x} w(x) \in \operatorname{RV}(\alpha)$ and assume that $\alpha>-1$. Then the subordinated density $g$ satisfies $g * g(x) / g(x) \rightarrow 2$.

Proof. We have $g * g(x)=\sum_{n=1}^{\infty}(p * p)_{n} w^{* n}(x)$. By assumption, $\left(p_{n}\right) \in \mathrm{SS}$. For $\epsilon \in(0,1)$, we can find $K$ such that

$$
2(1-\epsilon) p_{n} \leqslant(p * p)_{n} \leqslant 2(1+\epsilon) p_{n}, \quad n \geqslant K .
$$

Now we have

$$
g * g(x)=\sum_{n=1}^{K}(p * p)_{n} w^{* n}(x)+\sum_{n=K+1}^{\infty}(p * p)_{n} w^{* n}(x)=A+B .
$$

For the second term $B$, we have

$$
2(1-\epsilon) \sum_{n=K+1}^{\infty} p_{n} w^{* n}(x) \leqslant B \leqslant 2(1+\epsilon) \sum_{n=K+1}^{\infty} p_{n} w^{* n}(x),
$$


and it follows that

$$
2(1-\epsilon)(g(x)-C) \leqslant B \leqslant 2(1+\epsilon)(g(x)-C),
$$

where $C=\sum_{n=1}^{K} p_{n} w^{* n}(x)$. Now we consider $A+C=\sum_{n=1}^{K}\left((p * p)_{n}+p_{n}\right) w^{* n}(x)$. Since $p_{n} \leqslant 1$ and $(p * p)_{n} \leqslant 1$, we have $A+C \leqslant 2 \sum_{n=1}^{K} w^{* n}(x)$. By assumption, $w \in \operatorname{SHF}(\beta, \alpha)$ and $\alpha>-1$. Using Theorem 3 for $n=1,2, \ldots, K$, we have that

$$
w^{* n}(x) \sim C(\alpha, n) w(x)(x h(x))^{n-1}, \quad x \rightarrow \infty .
$$

Hence we can find constants $M, x^{\circ}$ such that

$$
A+C \leqslant M w(x)(x h(x))^{K-1}, \quad x \geqslant x^{\circ} .
$$

It follows that

$$
\frac{A+C}{g(x)} \leqslant \frac{M w(x)(x h(x))^{K-1}}{\sum_{n=K+1}^{\infty} p_{n} w^{* n}(x)}=M \frac{1}{\sum_{n=K+1}^{\infty} p_{n} w^{* n}(x) / w(x)(x h(x))^{K-1}} .
$$

Now for each fixed $n \geqslant K+1$, as $x \rightarrow \infty$, we have

$$
\frac{w^{* n}(x)}{w(x)(x h(x))^{K-1}} \sim \operatorname{const} \frac{(x h(x))^{n-1}}{(x h(x))^{K-1}}=\operatorname{const}(x h(x))^{n-K} \rightarrow \infty .
$$

Using Fatou's lemma, we conclude that $\lim \sup (A+C) / g(x)=0$. This proves the result.

\subsection{Applications in queueing}

Example 1. Consider a $G I / M / 1$ queue where the interarrival times have d.f. $A(x)$ with mean $\mu_{A}$ and the service times exponentially distributed with mean $1 / \mu$. The traffic intensity is given by $\rho=\left(\mu \mu_{A}\right)^{-1}$. Let the Laplace transform of $A$ be denoted by $\widehat{A}(s)$, and let $p$ denote the root of $p=\widehat{A}(\mu-\mu p)$. In the case where $A$ has a density $a(x)$, Prabhu [15, Thm. 16 (I.1.13)] showed that the idle period density of the $G I / M / 1$ queue is given by

$$
h(x)=\mu \mathrm{e}^{\mu(1-p) x} \int_{x}^{\infty} \mathrm{e}^{-\mu(1-p) y} a(y) \mathrm{d} y, \quad x \geqslant 0 .
$$

Now suppose that $a(x)=\mathrm{e}^{-\beta x} f(x) \in \operatorname{SHF}(\beta, \alpha)$. In this case we have

$$
\mathrm{e}^{-\mu(1-p) y} a(y)=\mathrm{e}^{-(\mu(1-p)+\beta) y} f(y) \in \operatorname{SHF}(\mu(1-p)+\beta, \alpha) .
$$

Now we can use Proposition 2 or Corollary 1 to obtain that

$$
\int_{x}^{\infty} \mathrm{e}^{-\mu(1-p) y} a(y) \mathrm{d} y \sim \frac{\mathrm{e}^{-(\mu(1-p)+\beta) x} f(x)}{\mu(1-p)+\beta},
$$

and it follows that

$$
h(x) \sim \mu \frac{\mathrm{e}^{-\beta x} f(x)}{\mu(1-p)+\beta}=\frac{\mu}{\mu(1-p)+\beta} a(x) .
$$


Example 2. Consider a $M / G / 1$ queue where the service times have d.f. $B(x)$ with mean $\mu_{B}$ and the interarrival times exponentially distributed with mean $1 / \lambda$. The traffic intensity is given by $\rho=\lambda \mu_{B}$. The distribution of the stationary waiting time is given by (cf. Asmussen [2])

$$
G(x)=(1-\rho) \sum_{n=0}^{\infty} \rho^{n} B_{0}^{* n}(x),
$$

where $B_{0}(x)$ is the equilibrium distribution given by

$$
B_{0}(x)=\frac{1}{\mu_{B}} \int_{0}^{x} \bar{B}(t) \mathrm{d} t .
$$

The equilibrium density is given by $b_{0}(x)=\bar{B}(t) / \mu_{0}$. The tail of $G$ is given by

$$
\bar{G}(x)=(1-\rho) \sum_{n=1}^{\infty} \rho^{n} \overline{B_{0}^{* n}}(x) .
$$

Now assume that $b_{0}(x)=\mathrm{e}^{-\beta x} f(x)$ where $f \in \mathrm{RV}(\alpha)$ and $\alpha<-1$. Also, assume that $\rho \int_{0}^{\infty} f(t) \mathrm{d} t<1$.

Using Proposition 2, we have $\bar{B}_{0}(x) \sim b_{0}(x) / \beta=\bar{B}(x) /\left(\beta \mu_{B}\right)$. From Proposition 4 we also have that

$$
\frac{b_{0}^{* k}(x)}{b_{0}(x)} \leqslant K(1+\epsilon)^{k}\left(\int_{0}^{\infty} f(t) \mathrm{d} t\right)^{k-1} \quad \forall k \geqslant 1
$$

and that

$$
\frac{b_{0}^{* k}(x)}{b_{0}(x)} \rightarrow k\left(\int_{0}^{\infty} f(t) \mathrm{d} t\right)^{k-1} \quad \forall k \geqslant 1 .
$$

Taking integrals here, it follows that

$$
\frac{1-B_{0}^{* k}(x)}{1-B_{0}(x)} \leqslant K(1+\epsilon)^{k}\left(\int_{0}^{\infty} f(t) \mathrm{d} t\right)^{k-1}
$$

and

$$
\frac{1-B_{0}^{* k}(x)}{1-B_{0}(x)} \rightarrow k\left(\int_{0}^{\infty} f(t) \mathrm{d} t\right)^{k-1} .
$$

Using dominated convergence, we obtain that

$$
\frac{1-G(x)}{1-B_{0}(x)} \rightarrow(1-\rho) \sum_{k=1}^{\infty} k \rho^{k}\left(\int_{0}^{\infty} f(t) \mathrm{d} t\right)^{k-1}=\frac{(1-\rho) \rho \int_{0}^{\infty} f(t) \mathrm{d} t}{\left(1-\rho \int_{0}^{\infty} f(t) \mathrm{d} t\right)^{2}}
$$

or equivalently that

$$
\frac{1-G(x)}{1-B(x)} \rightarrow \frac{(1-\rho) \rho \int_{0}^{\infty} f(t) \mathrm{d} t}{\beta \mu_{B}\left(1-\rho \int_{0}^{\infty} f(t) \mathrm{d} t\right)^{2}}
$$




\section{Concluding remarks}

1. It may be interesting to study sequences $\left(u_{n}\right)$ such thath $\left(u^{* 2}\right)_{n} / u_{n} \rightarrow \infty$.

2. We plan to study more general sequences (and functions) of the form $u_{n}=\mathrm{e}^{-a(n)} f_{n}$ where $\left(f_{n}\right) \in \operatorname{RS}(\alpha)$ and $a(n) \rightarrow \infty$.

3. In the future we plan to study also the bivariate case. In the case of functions, for example, we assume that $w(x, y)=\mathrm{e}^{-\alpha x-\beta y} f(x, y), x, y, \alpha, \beta>0$, where $f$ is a bivariate (regularly varying) function. As in Lemma 1, we can prove that, for general functions $f$, we have $w^{* k}(x, y) \leqslant \mathrm{e}^{-\alpha x-\beta y}(x y)^{(k-2) / 2}(h(x, y))^{k / 2}$, $x, y \geqslant 0$, where $h(x, y)=\int_{0}^{x} \int_{0}^{y} f^{2}(u, v) \mathrm{d} u \mathrm{~d} v$.

Acknowledgment. The authors express their appreciation to the anonymous reviewers for their detailed reviews and suggestions, allowing the final version of the paper to be significantly improved.

\section{References}

1. J.M.P. Albin and M. Sunden, On the asymptotic behaviour of Lévy processes. Part 1: Subexponential and exponential processes, Stochastic Processes Appl., 119:281-304, 2009.

2. S. Asmussen, Applied Probability and Queues, 2nd ed., Springer, New York, 2003.

3. O.E. Barndorff-Nielsen, Processes of normal inverse Gaussian type, Finance Stoch., 2:41-68, 1998.

4. N.H. Bingham, C.M. Goldie, and J.L. Teugels, Regular Variation, Encycl. Math. Appl., Vol. 27, Cambridge Univ. Press, Cambridge, 1987.

5. S. Borak, A. Misiorek, and R. Weron, Models for heavy tailed asset returns, in Statistical Tools for Finance and Insurance, Springer, Berlin, Heidelberg, 2011, pp. 21-55.

6. J. Chover, P. Ney, and S. Wainger, Functions of probability measures, J. Anal. Math., XXVI:255-302, 1973.

7. E. Eberlein and U. Keller, Hyperbolic distributions in finance, Bernoulli, 1:281-299, 1995.

8. P. Embrechts and E. Omey, Functions of power series, Yokohama Math. J., 32:77-88, 1984.

9. S. Foss, D. Korshunov, and S. Zachary, An Introduction to Heavy-Tailed and Subexponential Distributions, Springer Ser. Oper. Res. Financ. Eng., Springer, New York, 2011.

10. J.L. Geluk and L. de Haan, Regular Variation, Extensions and Tauberian Theorems, CWI Tracts, Vol. 40, Centrum voor Wiskunde en Informatica, Amsterdam, 1987.

11. B.G. Hansen and E. Willekens, The generalized logarithmic series distribution, Stat. Probab. Lett., 9:311-316, 1990.

12. C. Klüppelberg, Subexponential distributions and characterizations of related classes, Probab. Theory Relat. Fields, 82:259-269, 1989.

13. D.V. Lindley, Fiducial distributions and Bayes' theorem, J. R. Stat. Soc., Ser. B, 20:102-107, 1958.

14. S. Nadarajah, H.S. Bakouch, and R. Tahmasbi, A generalized Lindley distribution, Sankhyā, Ser. B, 73:331-359, 2011.

15. N.U. Prabhu, Stochastic Storage Models: Queues, Insurance Risk, Dams, and Data Communication, 2nd ed., Springer, 1998.

16. K. Prause, The Generalized Hyperbolic Model: Estimation, Financial Derivatives, and Risk Measures, PhD thesis, Freiburg University, 1999, available from: http://www. freidok. uni-freiburg.de/volltexte/15.

17. H. Schmidli, Compound sums and subexponentiality, Bernoulli, 5(6):999-1012, 1999. 
18. W. Schoutens, Lévy Processes in Finance, Wiley, New York, 2003.

19. V. Seshadri, Halphen's laws, in S. Kotz, C.B. Read, and D. L. Banks (Eds.), Encyclopedia of Statistical Sciences, Update Vol. 1, Wiley, New York, 1997, pp. 302-306.

20. H. Xu, M. Scheutzow, and Y. Wang, On a transformation between distributions obeying the principle of a single big jump, J. Math. Anal. Appl., 430:672-684, 2015.

21. H. Zakerzadeh and A. Dolati, Generalized Lindley distribution, J. Math. Ext., 3(2):13-25, 2009. 